\title{
(2) OPEN ACCESS \\ Silicone oil versus gas tamponade for primary rhegmatogenous retinal detachment treated successfully with a propensity score analysis: Japan Retinal Detachment Registry
}

\author{
Ryoh Funatsu, ${ }^{1}$ Hiroto Terasaki, ${ }^{1}$ Chihaya Koriyama, ${ }^{2}$ Toshifumi Yamashita, \\ Hideki Shiihara, ${ }^{1}$ Taiji Sakamoto (1) ${ }^{1}$
}

\begin{abstract}
- Additional supplemental material is published online only. To view, please visit the journal online (http://dx.doi. org/10.1136/bjophthalmol2021-319876)
\end{abstract}

'Ophhtalmology, Kagoshima University Graduate School of Medicine and Dental Sciences, Kagoshima, Japan

${ }^{2}$ Epidemiology and Preventive Medicine, Kagoshima

University Graduate School of Medicine and Dental Sciences, Kagoshima, Japan

\section{Correspondence to}

Dr Taiji Sakamoto Ophhtalmology, Kagoshima University Graduate School of Medicine and Dental Sciences, Kagoshima, Japan; tsakamot@m3.kufm. kagoshima-u.ac.jp

Received 13 June 2021 Accepted 25 July 2021
A) Check for updates

(c) Author(s) (or their employer(s)) 2021. Re-use permitted under CC BY-NC. No commercial re-use. See rights and permissions. Published by BMJ.

To cite: Funatsu $\mathrm{R}$

Terasaki H, Koriyama C, et al. Br J Ophthalmol Epub ahead of print: [please include Day Month Year]. doi:10.1136/ bjophthalmol-2021-319876

\begin{abstract}
Background/aims To compare the effects of silicone oil tamponade (SOT) to that of gas tamponade (GT) on the best-corrected visual acuity (BCVA) after successful vitrectomy for retinal detachment (RD).

Methods A retrospective, multicentre, nationwide study with RD who were registered in the Japan-RD Registry. All cases with RD treated with successful vitrectomy between February 2016 and March 2017 were studied. A propensity score matching was performed using the preoperative findings as covariates to adjust the relevant confounders. The primary outcome was the estimated mean difference of the postoperative BCVA in 6 months between eyes treated with SOT to those treated with GT. Results Of the 3446 cases registered, 2097 cases met the entry criteria. There were 2042 eyes that had GT and 55 eyes that had SOT. Primary success was defined as a reattached retina with no tamponade at 6 months. After propensity score matching, each group contained 40 cases. The preoperative BCVA was $0.966 \pm 0.738 \log M A R$ units in the GT group and 1.270 $\pm 0.945 \log M A R$ units in the SOT group $(p=0.177)$. Six months postoperatively, the BCVA in the GT group was significantly better at 0.309 logMAR units in the GT group than the 0.671 $\log M A R$ units in the SOT group $(p=0.002)$.

Conclusions Even after successful surgery for RD, eyes that experienced SOT had poorer BCVA than eyes treated with GOT. SOT should be considered cautiously.

Precis Propensity score analysis of eyes with rhegmatogenous RD showed that postoperative vision was worse in eyes treated once with silicone oil than with gas even after completely successful surgery.
\end{abstract}

\section{INTRODUCTION}

Silicone oil tamponade (SOT) is widely used to treat eyes with a severe retinal detachment (RD), and it has been reported to be equally or more effective than gas tamponade (GT).$^{1-8}$ Nonetheless, this does not necessarily mean that silicone oil is perfectly safe because there are reports that SOT can cause complications such as cataracts, glaucoma and paracentral dark spots. ${ }^{34-12}$ In addition, it was recently reported that the quality of silicone oil medical products varied from batch to batch even under the same brand name, and that they may be toxic. ${ }^{13-15}$ It has also been reported that unexplained visual impairments occurred from damages to retinal structures in eyes treated with SOT. ${ }^{16-18}$ There are also reports that eyes with non-complex RD treated by SOT had poorer postoperative retinal sensitivity and visual acuity than eyes treated by GT. ${ }^{19} 20$ Thus, there is a concern that the toxicity of silicone oil may be greater than that reported.

Randomised clinical trial (RCT) is theoretically the best way to answer questions about the efficacy of a specific treatment for a specific disease. However, RCTs comparing GT and SOT are not practical because the low incidence of SOT usage makes it difficult to recruit a sufficiently number of participants treated with SOT. It has also been pointed out that surgeons and patients have preferences, so that RCTs comparing existing surgical methods are not practical. $^{2122}$ Also, the huge cost of RCTs and their long duration required to obtain results are big problems.

In recent years, propensity score analyses using registry data have been conducted to overcome the shortcomings of RCTs. Although it is not possible to match unmeasured background factors as in RCTs, it has the advantage of being able to answer clinical questions with far less time and cost.

Thus, the purpose of this study was to compare the best-corrected visual acuity (BCVA) at 6 months after eyes with RD were treated by GT or SOT. In order to omit the effect of the difference in the power of GT and SOT to repair RD, the analysis was restricted to only those eyes where the surgery was successful and the tamponade material was removed. To accomplish this, we conducted a propensity score analysis of a multicentre database gathered by the Japanese Retina Vitreous Society (JRVS).

\section{METHODS}

\section{Data registry}

A detailed description of the study design has been published elsewhere. $^{2324}$ In short, every case of RD from 26 ophthalmological institutions in Japan were collected by a database project. All surgeries were performed by retinal specialists certified by the Japanese Ophthalmological Society. The registry data were collected online, between February 2016 and May 2017.

This study was approved by the Ethics Committee of the Kagoshima University (140093, 28-38), and the procedures conformed to the tenets of 


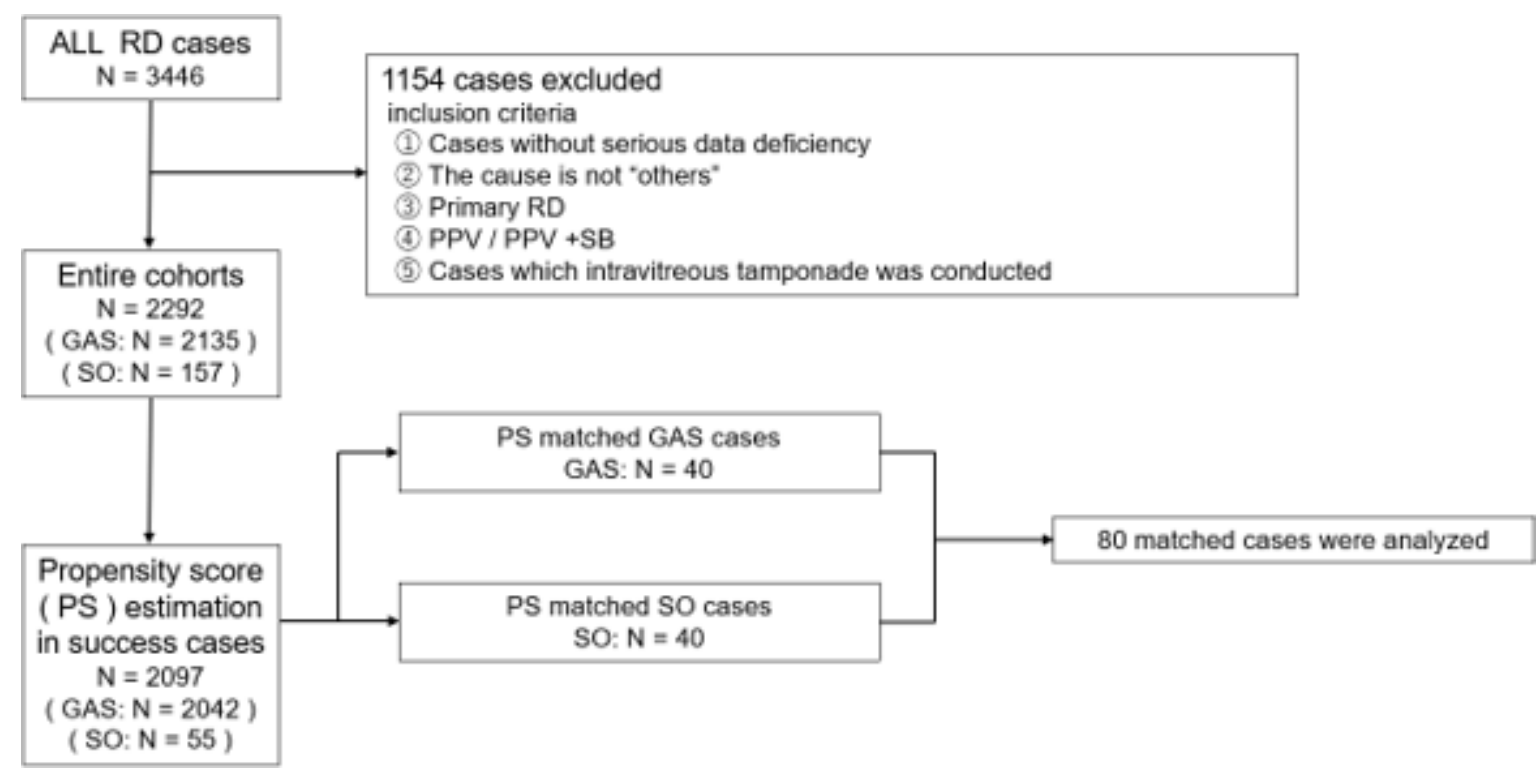

Figure 1 Flowchart describing selecting the study population. PPV, pars plana vitrectomy; PS, propensity score; RD, retinal detachment; SB, scleral buckling; SO, silicone oil.

the Declaration of Helsinki. The information collected was not personally identifiable, and details of obtaining research consent have been published. ${ }^{23}$

\section{Study participants}

The demographics of the patients have been published in detail elsewhere. ${ }^{23}$ The total number of cases of RD in the database was 3446. From these, we extracted cases with initial surgery for the RD by pars plana vitrectomy (PPV) or PPV with scleral buckling (SB) and an intravitreal tamponade. From these, 2292 cases were selected as the entire cohort by excluding cases with incomplete data, data entry errors or data that could not be analysed. The propensity score was calculated by selecting 2097 cases with 2042 eyes treated by GT and 55 eyes treated by SOT with successful results at 6 months after the surgery (figure 1). The important variables were selected by the JRVS registry committee members based on the previous reports. ${ }^{7825}$ A total of $50+$ items were collected as reported. ${ }^{2324}$ Complex or severe RDs were defined based on the EVRS study. ${ }^{7825}$ Postoperative data were collected for up to 6 months after the surgery and were registered by the individual surgeons.

\section{Definitions of primary success and failure}

The level of success and failure of the initial surgery were graded 1 to 3 at 6 months postoperatively as defined by the EVRS study. ${ }^{7825}$ The surgeons performed silicone oil removal at their discretion. Primary success was defined as no additional surgery or removable of silicone oil at the 6 postoperative months. In other words, in the case with retina reattached at 6 months after surgery, if the only additional surgery was silicone oil removal, we define it as success. The degree of failure was graded into three levels. Level 1 failure was defined as those eyes with the retina reattached after additional surgery before 6 months. This additional surgery did not include removal of the silicone oil, and the cases with redetachment after silicone oil removal and which require further surgery to reattach the retina at 6 months after primary surgery were included in level 1 failure. Failure level 2 was defined as persistent SOT at 6 months after the initial surgery with or without additional surgery. Failure level 3 was defined as a retina not reattached at 6 months after the initial surgery with or without additional surgery. ${ }^{25}$

\section{Visual function in primary success cases}

There was a significant trend towards the use of SOT for more severe cases at the initial diagnosis in this database. ${ }^{24}$ Also, it has been reported that silicone-filled eyes tended to have poorer postoperative BCVA even if the retina had been securely reattached. ${ }^{26}$ Therefore, we compared only the primary success cases of SOT and GT eyes. We compared the BCVA of these eyes at 6 months with alignment of the preoperative conditions using the following method. The BCVA at 1 month and 3 months after surgery was not tested for comparison because silicone oil was not removed in some cases during this period. Removing the silicone oil is known to cause unexplained vision loss. ${ }^{192027}$ To eliminate the effects of these cases, we compared the visual acuity at 1 month to that at 6 months. If visual acuity decreased by 0.2 $\log$ MAR units or more (10 early treatment diabetic retinopathy study (ETDRS) letters or more) from 1 month to 6 months for no apparent reason, we removed such cases from the statistical analyses.

\section{Statistical analyses}

The percentage of missing values for each variable was examined (online supplemental table S1). The missing values were resolved by using multiple imputations by chaining equations. The variables used for the completion of the missing values are shown in online supplemental table S1. Five completed data sets were created, and the following information was obtained: sex, age, prior eye surgery, number of days from the onset of signs and symptoms of RD to the date of surgery, preoperative intraocular pressure, preoperative BCVA, axial length of the eye, preoperative lens status, number of retinal breaks, type of retinal break, maximum size of retinal break, location of the retinal break, presence of a complex RD, presence of a macular detachment, presence of posterior vitreous detachment, presence of giant tear, presence of choroidal detachment, stage of proliferative vitreoretinopathy, quadrant of $\mathrm{RD}$ and presence of combined 
cataract surgery. The propensity score of the probability of using SOT was calculated using a multiple logistic regression model.

The means, SD and percentages of the patients' background data before the propensity score matching were calculated, and the significance of the differences between the GT and SOT groups was determined by Mann-Whitney $U$ tests, $\chi^{2}$ tests and Fisher's exact tests.

We performed propensity score matching for the GT and SOT groups using the data sets we created for each. Specifically, we used 1:1 pairing to eliminate bias in the number of samples between the two groups, and non-restorative matching to ensure that there was a sufficient large number of cases. To exclude pairs with widely separated propensity scores, a nearest neighbour matching was used with calliper set to 0.1 .

The balance of the background of the patients between the two groups after propensity score matching was assessed by the distribution of the propensity scores in each group and the Mann-Whitney $U$ tests for continuous variables or the Yatescorrected $\chi^{2}$ tests or Fisher's exact tests for qualitative variables. After matching, the means and SD were calculated for the visual acuity at 1, 3 and 6 months after the surgery, and the visual acuity at 6 months after the surgery was compared between the two groups using the Mann-Whitney U tests.

Sensitivity analysis was performed to evaluate the robustness of the results in the group from which the primary success group was extracted. Multiple linear regression analysis with the propensity score and the tamponade substance as independent variables and 6-month postoperative visual acuity as dependent variable and inverse probability treatment weighting were used to analyse the average treatment effect on the treated group. The robustness of the results was confirmed. The R software (V.4.0.5) was used for all analyses with $p$ values $<0.05$ as cut-off value.

\section{RESULTS \\ Preoperative characteristics of patients before propensity score matching}

Of the 2097 collected cases, 2042 cases were GT cases and 55 were SOT cases. There were significantly more men $(\mathrm{p}<0.001)$, atopic, macular hole RD with severe myopia and traumatic detachment RD $(\mathrm{p}<0.001)$ in the SOT group. In addition, there were fewer cases of combined cataract surgery $(p=0.025)$ and more cases of SB in the SOT group $(p=0.008$; table 1). The SOT group had significantly lower preoperative intraocular pressure $(\mathrm{p}<0.001)$, poorer preoperative visual acuity $(\mathrm{p}<0.001)$, longer axial length $(\mathrm{p}=0.016)$ and a higher percentage of complicated RD $(p<0.001)$ cases. The rates of giant tears, macular detachments, choroidal detachments, RD size and PVR were also higher in the SOT group (online supplemental table S2). There was a smaller percentage of retinal tear in the types of retinal breaks $(p<0.001)$. In addition, there were more retinal breaks $(p=0.026)$, more inferior localisation $(p<0.001)$ and bigger size $(p=0.008)$ of the largest retinal break in the SOT group (online supplemental table S3). A simple comparison of these groups showed that the SOT group had significantly poorer BCVA at 6 months $(p=0.002$, online supplemental table S4).

\begin{tabular}{|c|c|c|c|c|}
\hline & & $\begin{array}{l}\text { Gas }^{*} \\
(n=2042)\end{array}$ & $\begin{array}{l}\text { Silicone oil } \\
(\mathrm{n}=55)\end{array}$ & $\begin{array}{l}\text { P } \\
\text { value }^{\S}\end{array}$ \\
\hline Age (years) & & $59.69 \pm 11.61$ & $59.04 \pm 17.65$ & 0.373 \\
\hline \multirow[t]{3}{*}{ History type } & None & $1608(78.75 \%)$ & $36(65.45 \%)$ & 0.017 \\
\hline & $\begin{array}{l}\text { Vitrectomy excluding retinal detachment } \\
\text { surgery }\end{array}$ & $18(0.88 \%)$ & $2(3.64 \%)$ & \\
\hline & Photocoagulation for retinal break & $43(2.11 \%)$ & $0(0.00 \%)$ & \\
\hline \multirow[t]{7}{*}{ RRD type } & Related to posterior vitreous detachment & $1699(83.20 \%)$ & $30(54.55 \%)$ & $<0.001$ \\
\hline & Related to atrophic hole & $143(7.00 \%)$ & $4(7.27 \%)$ & \\
\hline & Related to atopic dermatitis & $30(1.47 \%)$ & $5(9.09 \%)$ & \\
\hline & $\begin{array}{l}\text { Retinal detachment with macular hole } \\
\text { related to myopia }\end{array}$ & $52(2.55 \%)$ & $5(9.09 \%)$ & \\
\hline & Related to intraocular surgery & $57(0.28 \%)$ & $3(5.45 \%)$ & \\
\hline & Hereditary & $6(0.29 \%)$ & $1(1.82 \%)$ & \\
\hline & Traumatic & $41(2.01 \%)$ & $7(12.73 \%)$ & \\
\hline \multirow[t]{3}{*}{ Lens status } & Phakia & $406(19.88 \%)$ & $15(27.27 \%)$ & 0.282 \\
\hline & Aphakia & $0(0.00 \%)$ & $0(0.00 \%)$ & \\
\hline & Pseudophakia & $1636(80.12 \%)$ & $40(72.73 \%)$ & \\
\hline \multicolumn{2}{|l|}{ Onset to surgery (days) } & $12.60 \pm 34.76$ & $34.05 \pm 70.91$ & 0.017 \\
\hline Type of surgery & With scleral buckling & $92(4.51 \%)$ & $20(36.36 \%)$ & 0.008 \\
\hline Combined cataract surgery & + & $1389(68.02 \%)$ & $29(52.72 \%)$ & 0.025 \\
\hline \multicolumn{5}{|c|}{ 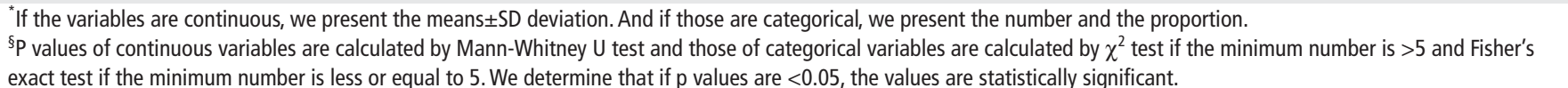 } \\
\hline
\end{tabular}




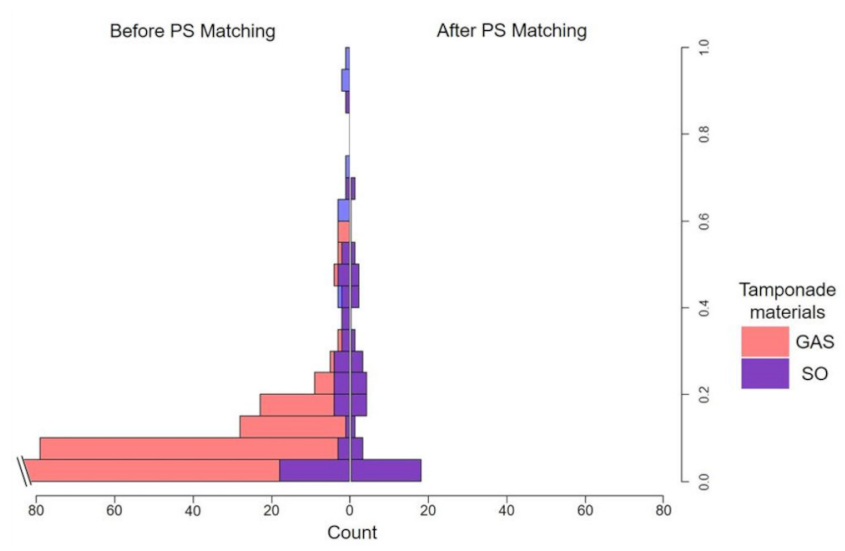

Figure 2 Distribution of propensity score before and after propensity score matching. Doublet line is used for a broken bar due to scale difference. PS, propensity score; SO, silicone oil.

\section{Preoperative characteristics of patients in two groups after propensity score matching}

The distribution of the propensity scores before and after matching is shown in figure 2. After matching, the sample size was 40 cases each in the GT and SOT groups (table 2). Examination of the background factors among the tamponade materials, the differences in the BCVA had the smallest $\mathrm{p}$ values (table 3, $\mathrm{p}=0.177$ ) (see table 3, online supplemental table S5).

\section{BCVA after 6 months}

In SOT group, the mean durations to silicone oil removal were $3.2 \pm 1.3$ months. The mean BCVA of the SOT group and the GT group after matching was $0.898 \pm 0.579 \log$ MAR units (Snellen 20/158) and 0.448 \pm 0.510 logMAR units (Snellen 20/56), $0.758 \pm 0.588 \log$ MAR units (Snellen 20/115) and 0.334 \pm 0.451 logMAR units (Snellen 20/43) and 0.671 $\pm 0.606 \log$ MAR units (Snellen 20/94) and 0.309 $\pm 0.513 \log$ MAR units (Snellen 20/41) at 1 month, 3 months and 6 months after the surgery, respectively. The BCVAs were significantly better in the GT group at 6 months ( $p=0.002$, table 4$)$.

There were four eyes that had a poorer vision after the silicone oil was removed. After this restriction, the SOT group had a mean BCVA of $0.597 \operatorname{logMAR}$ units (Snellen 20/79), which was $0.288 \log$ MAR units (Snellen 20/39) significantly worse than the GT eyes $(p=0.009$; online supplemental table $S 6)$.

\section{Sensitivity analyses}

Sensitivity analyses were performed by multiple regression analysis and inverse probability treatment weighting to confirm the robustness of the results. The visual acuity at 6 months postoperatively was significantly worse in the SOT group than in the GT group whether multiple regression analysis was performed using propensity scores or inverse probability treatment weighting (online supplemental tables S7 and S8). The results obtained by these methods were equal to those obtained by propensity score matching (figure 3).

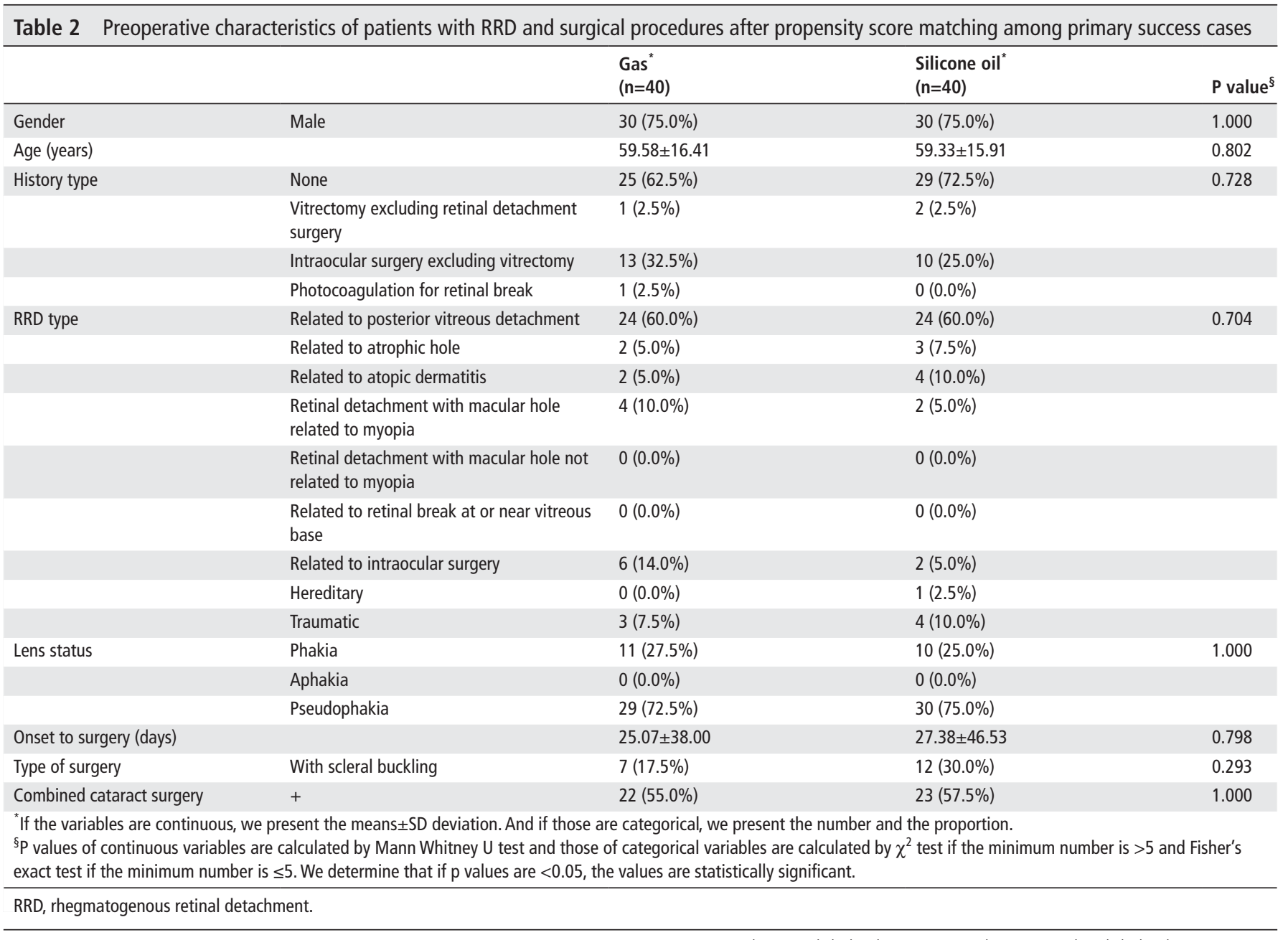


Table 3 Preoperative ocular findings of patients with rhegmatogenous retinal detachment after propensity score matching among primary success cases

\begin{tabular}{|c|c|c|c|c|}
\hline & & $\begin{array}{l}\text { Gas* } \\
(n=40)\end{array}$ & $\begin{array}{l}\text { Silicone oil* } \\
(n=40)\end{array}$ & $\begin{array}{l}P \\
\text { valuet }\end{array}$ \\
\hline $\begin{array}{l}\text { Intraocular pressure } \\
(\mathrm{mm} \mathrm{Hg})\end{array}$ & & $11.22 \pm 4.67$ & $11.05 \pm 4.25$ & 0.952 \\
\hline $\begin{array}{l}\text { Best corrected visual } \\
\text { acuity (logMAR) }\end{array}$ & & $0.966 \pm 0.738$ & $1.270 \pm 0.945$ & 0.177 \\
\hline $\begin{array}{l}\text { Best-corrected visual } \\
\text { acuity }<0.8 \text { logMAR } \\
\text { units }\end{array}$ & + & $17(42.50 \%)$ & $14(35.00 \%)$ & 0.646 \\
\hline $\begin{array}{l}\text { Axial length } \\
(\mathrm{mm})\end{array}$ & & $26.00 \pm 2.64$ & $26.68 \pm 3.04$ & 0.489 \\
\hline $\begin{array}{l}\text { Spherical equivalent } \\
\text { (diopter) }\end{array}$ & & $-2.73 \pm 5.35$ & $-3.05 \pm 5.17$ & 0.979 \\
\hline $\begin{array}{l}\text { Posterior vitreous } \\
\text { detachment }\end{array}$ & + & $32(80.0 \%)$ & $32(80.0 \%)$ & 1.000 \\
\hline $\begin{array}{l}\text { Complex retinal } \\
\text { detachment }\end{array}$ & + & $31(77.5 \%)$ & $28(70.0 \%)$ & 1.000 \\
\hline Giant tear & + & $8(20.0 \%)$ & $10(25.0 \%)$ & 0.789 \\
\hline Macula detachment & + & $33(82.5 \%)$ & $34(85.0 \%)$ & 1.000 \\
\hline $\begin{array}{l}\text { Choroidal } \\
\text { detachment }\end{array}$ & + & $6(15.0 \%)$ & $7(17.50 \%)$ & 1.000 \\
\hline \multirow{4}{*}{$\begin{array}{l}\text { Area of retinal } \\
\text { detachment }\end{array}$} & One quadrant & $8(20.0 \%)$ & $3(7.5 \%)$ & 0.424 \\
\hline & Two quadrants & $16(40.0 \%)$ & $17(42.5 \%)$ & \\
\hline & $\begin{array}{l}\text { Three } \\
\text { quadrants }\end{array}$ & $6(15.0 \%)$ & $9(22.5 \%)$ & \\
\hline & $\begin{array}{l}\text { Four } \\
\text { quadrants }\end{array}$ & $10(25.0 \%)$ & $11(27.5 \%)$ & \\
\hline \multirow{3}{*}{$\begin{array}{l}\text { Stage of proliferative } \\
\text { vitreoretinopathy }\end{array}$} & $\mathrm{N} / \mathrm{A}$ & $17(42.5 \%)$ & $22(55.0 \%)$ & 0.183 \\
\hline & B & $10(25.0 \%)$ & $12(30.0 \%)$ & \\
\hline & C & $13(32.5 \%)$ & $6(15.0 \%)$ & \\
\hline
\end{tabular}

*If the variables are continuous, we present the means \pm SD deviation. And if those are categorical, we present the number and the proportion.

tP values of continuous variables are calculated by Mann-Whitney $U$ test and those of categorical variables are calculated by $\chi^{2}$ test if the minimum number is $>5$ and Fisher's exact test if the minimum number is $\leq 5$. We determine that if $p$ values are $<0.05$, the values are statistically significant.

\section{DISCUSSION}

Our results showed that RD eyes that were reattached after 6 months with SOT had significantly poorer BCVA than the eyes with GT (online supplemental table S4). This might be because more severe RDs, such as those with PVR grade C or giant tear, were in SOT group (table 1, online supplemental table S2). Since there were reports that SOT group showed a lower anatomical success rate than the GT group and the $15 \%$ of eyes with silicone oil removal showed redetachment, we restricted the cases that silicone oil was removable within 6 months postoperatively and did not have redetachment. ${ }^{28}{ }^{29}$ Furthermore, as there are cases

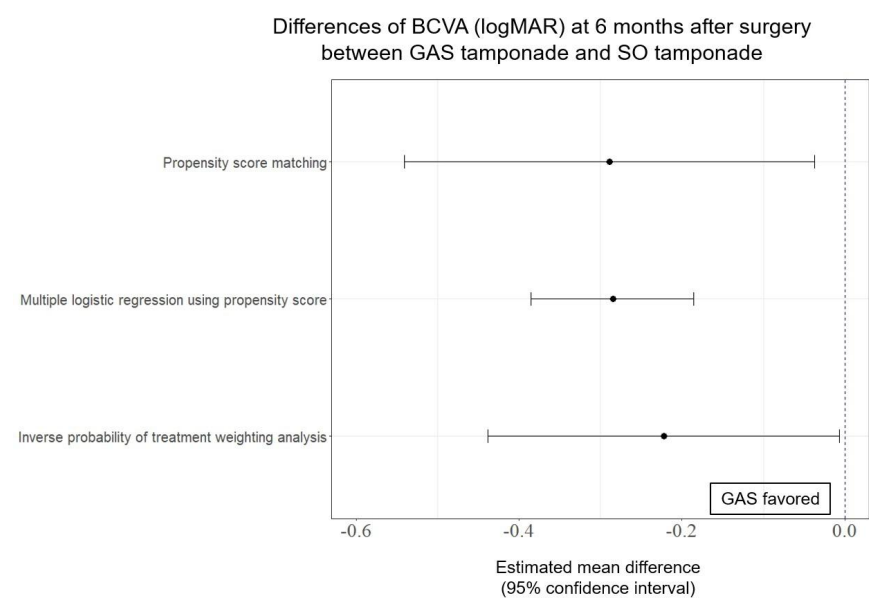

Figure 3 Forest plot describing the estimated mean difference of bestcorrected visual acuity at 6 months postoperatively for each analysis method. BCVA, best corrected visual acuity; SO, silicone oil.

in which simply removing the silicone oil causes a decrease in the $\mathrm{BCVA},{ }^{16}{ }^{17}$ we excluded four cases in which the BCVA decreased from 1 month to 6 months after the removal of silicone oil to exclude this effect. Nevertheless, the BCVA of the SOT group was still significantly poorer. In addition, multiple linear regression analysis using propensity scores and inverse probability treatment weighting estimations found that the results were similar confirming that these results were not obtained by chance but were rather robust.

Why the SOT eyes had the poorer vision was not determined. There have been studies reporting the presence of retinal cysts, thinning of the retina and central scotoma in silicone-filled eyes, and these findings have been reported as factors that deteriorate postoperative visual acuity. ${ }^{19203031}$ However, we could not determine the exact reason because we did not have such data in this registry.

Comparing the GT group to the SOT group in RD eyes by RCTs would allow comparisons of the outcomes. However, the effects of silicone oil on vision would be masked by the influence of preoperative eye conditions and outcomes. Most importantly, there were not enough cases to match the backgrounds in a short period of time. In addition, RCTs on surgical procedures, where the surgeons and patients already have preferences, make RCTs not only difficult to perform but also unethical. ${ }^{21}$ Propensity score matching studies using real-world data have been recommended for the evaluation of such conditions. ${ }^{22}$

Currently, SOT is mainly used to treat severe retinal diseases, but they may also be used for relatively mild cases, for example, patients with only one eye, needing flight travel and those who cannot maintain a specific posture. In this study, the proportion of mild cases, such as the case with preoperative BCVA of better than $0.8 \log$ MAR units, was $35 \%$ in the SOT group (table 3). It

Table 4 Comparing postoperative outcomes of patients with rhegmatogenous retinal detachment between tamponade materials after propensity score matching among primary success cases

\begin{tabular}{lllll}
\hline & & Gas $^{*}(\mathrm{n}=40)$ & Silicone oil* $(\mathrm{n}=40)$ & P valuet \\
\hline Best corrected visual acuity (logMAR) & First month & $0.448 \pm 0.510$ & $0.898 \pm 0.579$ & - \\
& Third month & $0.334 \pm 0.451$ & $0.758 \pm 0.588$ & - \\
& Sixth month & $0.309 \pm 0.513$ & $0.671 \pm 0.606$ & 0.002 \\
\hline
\end{tabular}

*If the variables are continuous, we present the means \pm SD deviation.

$\mathrm{tP}$ values of continuous variables are calculated by Mann-Whitney $U$ test. We determine that if $p$ values are $<0.05$, the values are statistically significant. 
may be necessary to refrain from the use of silicone oil in RD eyes considering the present results.

The results of an RCT that compared GT to SOT in eyes with diabetic retinopathy were recently reported. ${ }^{32}$ The results showed that the postoperative BCVA was poorer in the SOT eyes than the GT eyes at 6 months. These data are comparable to our results. That study was limited to cases with diabetic retinopathy and tractional $\mathrm{RD}$ with preoperative visual acuity of $1.75 \operatorname{logMAR}$ units (Snellen 20/1125) in GT eyes and 1.86 logMAR units (Snellen 20/1149) in the SOT eyes. The comparable preoperative BCVAs in our study were 0.96 logMAR units (Snellen 20/182) in the GT eyes and $1.26 \log$ MAR units (Snellen 20/364) in the SOT eyes. Thus, their subjects had poorer preoperative BCVA than our cases. The average period from the onset to the symptoms and signs of RD to the time of surgery was $>11$ months in their study, which is longer than that in our registry in which $89.8 \%$ of our patients underwent surgery within 1 month after the onset. It is possible that their cases would be more vulnerable to the insults of surgery or tamponade than our cases.

The results of RCT provide important evidence in general, but the interpretation of the results is difficult. It is known that the results are applicable only to those cases that meet the same criteria under similar conditions. Thus, in choosing the tamponade material in $\mathrm{RD}$, it is necessary to consider each condition with reference to both the earlier and our results.

One of the advantages of the present study is the far shorter time to reach a conclusion than RCT. Because this study used an established database registry, ${ }^{23} 2433$ it required only 1 week to obtain the first results. It took 2.5 years to collect the data for the RCT mentioned earlier, ${ }^{33}$ and the difference was significant. In addition, it has become too expensive to organise a wellpowered RCT. The present analysis could be completed even without any strong financial support. More importantly, RCTs are now considered to be experimental in nature and should not expose patients to needless risks. ${ }^{21} 2234$ Studies using registries rarely raise serious ethical issues. The data were from 128 surgeons nationwide and have the advantage of generalising the results.

This study also has limitations. First, this was a retrospective observational study and there is a possibility that unmeasured confounding factors may not have been adjusted for. This is an inevitable problem of propensity score matching analysis. It is also possible that the number of cases in which SOT was chosen was small in the registry data, which could have created a bias. Propensity matching is suitable for the analysis of such rare diseases or rare conditions. Considering the rarity of SOT among all RD cases, the present approach should be reasonable. Furthermore, as the database did not contain information on optical coherence tomography and defined methods for silicone oil removal, such as whether the removal was performed manually with a syringe, automatically with a vitreous machine or with fluid-air replacement, we cannot eliminate the possibility that they may have affected the results. This is also a limitation of this study.

In conclusion, the propensity score analysis of the real-world data showed that SOT may be related to a poorer postoperative vision than eyes treated with GT even after the completely successful surgery. In cases of RD where the use of SOT is being considered, the use of SOT should be selected very cautiously. More specifically, careful consideration for the use of SOT should be made especially in eyes with possible damaged retina, such as glaucoma, diabetic retinopathy or optic neuropathy. In future studies, it will be necessary to construct a database that includes information on the optical coherence tomography findings and the condition of the fellow eyes.

Collaborators The authors thank Professor Emeritus Duco Hamasaki of Bascom Palmer Eye Institute, University of Miami, FL, for providing critical discussions and suggestions to our study and editing of the final manuscript. List of surgeons in J-RD Registry Project institutions and their representatives. 1. Chiba University: Shuichi Yamamoto, Takayuki Baba, Eiju Sato, Masayasu Kitahashi, Tomoaki Tatsumi, Gen Miura, Tomohiro Niizawa. 2. Kagoshima University: Taiji Sakamoto, Keita Yamakiri, Toshifumi Yamashita, Hiroki Otsuka, Seiji Sameshima, Narimasa Yoshinaga, Shozo Sonoda. 3. Kyorin University: Akito Hirakata, Takashi Koto, Makoto Inoue, Kazunari Hirota, Yuji Itoh, Tadashi Orihara, Yoshinobu Emoto, Masahiko Sano, Hiroyuki Takahashi, Ryo Tokizawa. 4. Yamagata University: Hidetoshi Yamashita, Koichi Nishitsuka, Yutaka Kaneko, Katsuhiro Nishi. 5. Asahikawa Medical University Hospital: Akitoshi Yoshida, Shinji Ono, Hiroyuki Hirokawa, Kenji Sogawa, Tsuneaki Omae, Akihiro Ishibazawa. 6. Gunma University: Shoji Kishi, Hideo Akiyama, Hidetaka Matsumoto, Ryo Mukai, Masahiro Morimoto. 7. Hirosaki University: Mitsuru Nakazawa, Yukihiko Suzuki, Takashi Kudo, Kobu Adachi. 8. Hokkaido University: Susumu Ishida, Kousuke Noda, Satoru Kase, Shohei Mori, Ryo Ando, Michiyuki Saito, Tomohiro Suzuki. 9. Kansai Medical University Hospital: Kanji Takahashi, Yoshimi Nagai, Tadashi Nakauchi, Haruhiko Yamada. 10. Kindai University Sakai Hospital: Shunji Kusaka, Daishi Tsujioka. 11. Kyoto University: Akitaka Tsujikawa, Kiyoshi Suzuma. 12. Kyushu University: Tatsuro Ishibashi, Koh-Hei Sonoda, Yasuhiro Ikeda, Riichiro Kohno, Keijiro Ishikawa. 13. Mie University: Mineo Kondo, Maki Kozawa. 14. Nagasaki University: Takashi Kitaoka, Eiko Tsuiki. 15. Nagoya City University: Yuichiro Ogura, Munenori Yoshida, Hiroshi Morita, Aki Kato, Yoshio Hirano, Kazuhiko Sugitani. 16. Nagoya University: Hiroko Terasaki, Takeshi Iwase, Yasuki Ito, Shinji Ueno, Hiroki Kaneko, Norie Nonobe, Taro Kominami. 17. National Center for Child Health and Development: Noriyuki Azuma, Tadashi Yokoi. 18. Nihon University Hospital: Hiroyuki Shimada, Hiroyuki Nakashizuka, Takayuki Hattori, Ari Shinojima, Yorihisa Kitagawa. 19. Okayama University: Fumio Shiraga, Yuki Morizane, Shuhei Kimura. 20. Osaka Medical School: Tsunehiko Ikeda, Teruyo Kida, Takaki Sato, Masanori Fukumoto. 21. Osaka Rosai Hospital: Kazuyuki Emi, Hiroshi Nakashima. 22. Shiga Medical University: Masahito Ohji, Masashi Kakinoki, Osamu Sawada. 23. Takeuchi Eye Clinic: Shinobu Takeuchi, Sumiyoshi Tanaka. 24. Tokyo Women's Medical College: Tomohiro lida, Hideki Koizumi, Ichiro Maruko, Taiji Hasegawa, Akiko Kogure. 25. Yamanashi University: Hiroyuki lijima, Tomohiro Oshiro, Yasushi Tateno, Wataru Kikushima, Atsushi Sugiyama, Seigo Yoneyama. 26. Yokohama City University Medical Center: Kazuaki Kadonosono, Shimpei Sato, Shin Yamane.

Contributors RF and $\mathrm{HT}$ are joint first authors. RF and $\mathrm{HT}$ contributed equally to the study. Conception and design: RF, HT, TY, HS and TS. Analysis and interpretation: RF, HT and CK. Data collection: HT, TY, HS and TS. Critically revised the manuscript: RF, HT, CK and TS. Obtained funding: TS. Overall responsibility: RF, HT and TS.

Funding This study was supported by the JRVS and JSPS KAKENHI grant number $21 \mathrm{H03095}$. The sponsor or funding organisation had no role in the design or conduct of this research.

Competing interests None declared.

Patient consent for publication Not required.

Provenance and peer review Not commissioned; externally peer reviewed.

Data availability statement Data are available upon reasonable request. The datasets used during the current study are available from the corresponding author on request. The detailed protocol is published in our earlier paper. Sakamoto T, et al. Japan-Retinal Detachment Registry Report I: preoperative findings in eyes with primary retinal detachment. Jpn J Ophthalmol 2019 doi: 10.1007/s 10384- 01900702-6.

Supplemental material This content has been supplied by the author(s). It has not been vetted by BMJ Publishing Group Limited (BMJ) and may not have been peer-reviewed. Any opinions or recommendations discussed are solely those of the author(s) and are not endorsed by BMJ. BMJ disclaims all liability and responsibility arising from any reliance placed on the content. Where the content includes any translated material, BMJ does not warrant the accuracy and reliability of the translations (including but not limited to local regulations, clinical guidelines, terminology, drug names and drug dosages), and is not responsible for any error and/or omissions arising from translation and adaptation or otherwise.

Open access This is an open access article distributed in accordance with the Creative Commons Attribution Non Commercial (CC BY-NC 4.0) license, which permits others to distribute, remix, adapt, build upon this work non-commercially, and license their derivative works on different terms, provided the original work is properly cited, appropriate credit is given, any changes made indicated, and the use is non-commercial. See: http://creativecommons.org/licenses/by-nc/4.0/.

\section{ORCID iD}

Taiji Sakamoto http://orcid.org/0000-0003-0287-3801 


\section{REFERENCES}

1 Cibis PA, Becker B, Okun E, et al. The use of liquid silicone in retinal detachment surgery. Arch Ophthalmol 1962;68:590-9.

2 Lucke $\mathrm{KH}$, Foerster MH, Laqua H. Long-Term results of vitrectomy and silicone oil in 500 cases of complicated retinal detachments. Am J Ophthalmol 1987;104:624-33.

3 van Meurs JC, Mertens DA, Peperkamp E, et al. Five-Year results of vitrectomy and silicone oil in patients with proliferative vitreoretinopathy. Retina 1993;13:285-9.

4 Federman JL, Schubert HD. Complications associated with the use of silicone oil in 150 eyes after retina-vitreous surgery. Ophthalmology 1988;95:870-6.

5 Schwartz SG, Flynn HW, Wang X, et al. Tamponade in surgery for retinal detachment associated with proliferative vitreoretinopathy. Cochrane Database Syst Rev 2020:5:Cd006126.

6 Vitrectomy with silicone oil or sulfur hexafluoride gas in eyes with severe proliferative vitreoretinopathy: results of a randomized clinical trial. silicone study report 1. Arch Ophthalmol 1992;110:770-9.

7 Adelman RA, Parnes AJ, Sipperley JO, et al. Strategy for the management of complex retinal detachments: the European vitreo-retinal Society retinal detachment study report 2. Ophthalmology 2013;120:1809-13.

8 Adelman RA, Parnes AJ, Ducournau D, et al. Strategy for the management of uncomplicated retinal detachments: the European vitreo-retinal Society retinal detachment study report 1. Ophthalmology 2013;120:1804-8.

9 Chan C, Okun E. The question of ocular tolerance to intravitreal liquid silicone. A longterm analysis. Ophthalmology 1986;93:651-60.

10 Herbert EN, Habib M, Steel D, et al. Central scotoma associated with intraocular silicone oil tamponade develops before oil removal. Graefes Arch Clin Exp Ophthalmol 2006:244:248-52

11 Scheerlinck LM, Schellekens PA, Liem AT, et al. Incidence, risk factors, and clinical characteristics of unexplained visual loss after intraocular silicone oil for macula-on retinal detachment. Retina 2016;36:342-50.

12 Ghoraba HH, Zaky AG, Heikal MA, et al. Silicone Oil-Related visual loss. Ophthalmologica 2017;238:59-67.

13 Steel DHW, Wong D, Sakamoto T. Silicone oils compared and found wanting. Graefes Arch Clin Exp Ophthalmol 2020.

14 Mendichi R, Schieroni AG, Piovani D, et al. Comparative study of chemical composition, molecular and rheological properties of silicone oil medical devices. Trans/ Vis Sci Technol 2019:8:9.

15 Barca F, Caporossi T, Rizzo S. Silicone oil: different physical proprieties and clinical applications. Biomed Res Int 2014;2014:1-7.

16 Roca JA, Wu L, Berrocal M, et al. Un-explained visual loss following silicone oil removal: results of the pan American collaborative retina study (PACORES) group. Int $J$ Retina Vitreous 2017:3:26.

17 Cazabon Set al. Visual loss following removal of intraocular silicone oil. Br J Ophthalmol 2005:89:799-802.

18 Zhou Y, Zhang S, Zhou H, et al. Comparison of fundus changes following silicone oil and sterilized air tamponade for macular-on retinal detachment patients. BMC Ophthalmol 2020;20:249
19 Lee SH, Han JW, Byeon SH, et al. Retinal layer segmentation after silicone oil or gas tamponade for macula-on retinal detachment using optical coherence tomography. Retina 2018;38:310-9.

20 Raczyńska D, Mitrosz K, Raczyńska K, et al. The influence of silicone oil on the ganglion cell complex after pars plana vitrectomy for rhegmatogenous retinal detachment. Curr Pharm Des 2018;24:3476-93.

21 Lonjon G, Boutron I, Trinquart L, et al. Comparison of treatment effect estimates from prospective nonrandomized studies with propensity score analysis and randomized controlled trials of surgical procedures. Ann Surg 2014;259:18-25.

22 Farrokhyar F, Karanicolas PJ, Thoma A, et al. Randomized controlled trials of surgical interventions. Ann Surg 2010;251:409-16.

23 Sakamoto T, Kawano S, Kawasaki R, et al. Japan-Retinal detachment Registry report I: preoperative findings in eyes with primary retinal detachment. Jpn J Ophthalmol 2020;64:1-12.

24 Koto T, Kawasaki R, Yamakiri K, et al. Six-Months primary success rate for retinal detachment between vitrectomy and scleral buckling. Retina 2020;Publish Ahead of Print.

25 Adelman RA, Parnes AJ, Michalewska Z, et al. Clinical variables associated with failure of retinal detachment repair: the European vitreo-retinal Society retinal detachment study report number 4. Ophthalmology 2014;121:1715-9.

26 Smith RC, Smith GT, Wong D. Refractive changes in silicone filled eyes. Eye 1990:4:230-4.

27 Azen Set al. Silicone oil in the repair of complex retinal detachments a prospective observational multicenter study. Ophthalmology 1998;105:1587-97.

28 Abrams GW, Azen SP, McCuen BW, Gary W, Brooks W, et al. Vitrectomy with silicone oil or long-acting gas in eyes with severe proliferative vitreoretinopathy: results of additional and long-term follow-up. silicone study report 11. Arch Ophthalmol 1997; 115:335-44.

29 Hutton WL, Azen SP, Blumenkranz MS, et al. The effects of silicone oil removal. silicone study report 6. Arch Ophthalmol 1994;112:778-85.

30 Dormegny L, Jeanjean LC, Liu X, et al. Visual impairment and macular vascular remodeling secondary to retrograde maculopathy in retinal detachment treated with silicon oil tamponade. Retina 2021;41:309-16.

31 Shalchi Z, Mahroo OA, Shunmugam M, et al. Spectral domain optical coherence tomography findings in long-term silicone oil-related visual loss. Retina 2015:35:555-63.

32 Rush RB, Del Valle Penella A, et al. Silicone oil versus perfluoropropane gas tamponade during vitrectomy for tractional retinal detachment or fibrous proliferation: a randomized clinical trial. Retina 2020

33 Kawano S, Imai T, Sakamoto T, et al. Scleral buckling versus pars plana vitrectomy in simple phakic macula-on retinal detachment: a propensity score-matched, registrybased study. Br J Ophthalmol 2021. doi:10.1136/bjophthalmol-2020-318451. [Epub ahead of print: 29 Jan 2021].

34 James S, Rao SV, Granger CB. Registry-based randomized clinical trials--a new clinical trial paradigm. Nat Rev Cardiol 2015;12:312-6. 